\title{
Qualidade de vida e fatores associados em discentes de uma universidade pública do interior da Amazônia
}

\author{
Quality of life and associated factors among students at a public university in the interior of \\ the Amazon
}
Calidad de vida y factores asociados en estudiantes de una universidad pública del interior de la Amazonía

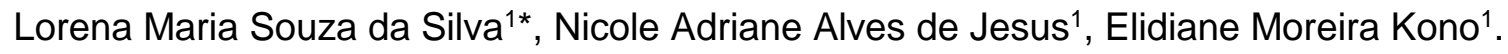

\begin{abstract}
RESUMO
Objetivo: Avaliar a qualidade de vida dos discentes do curso de fisioterapia de uma universidade pública no interior da Amazônia e fazer a comparação dos valores encontrados. Métodos: Estudo comparativo, transversal, quantitativo, descritivo, realizado com discentes do curso de fisioterapia do primeiro ao último ano de uma universidade pública do interior da Amazônia. Foi utilizado para avaliar a qualidade de vida desses discentes, o questionário WHOQOL-Bref. Resultados: Participaram da pesquisa 30 discentes de ambos os sexos do curso de Fisioterapia do $1^{\circ}, 3^{\circ}$ e $5^{\circ}$ ano de uma Universidade estadual no interior da Amazônia, os discentes responderam perguntas sobre os domínios: físicos, psicológicos, ambiente e relações sociais, no qual os que obtiveram o maior resultado de qualidade de vida foram os discentes do $5^{\circ}$ ano com um total de $57,21 \%$, em segundo o $3^{\circ}$ ano com $52,88 \%$ e por último o $1^{\circ}$ ano com $49,76 \%$. Conclusão: Os discentes do $1^{\circ}$ ano apresentaram a pior qualidade de vida por responderem que estão insatisfeitos com sua saúde física e mental, relações sociais e o ambiente quem vivem.
\end{abstract}

Palavras-chave: Qualidade de vida, Universitários, Saúde mental.

\begin{abstract}
Objective: To evaluate the quality of life of the students of the physiotherapy course at a public university in the interior of the Amazon and to compare the values found. Methods: Comparative, cross-sectional, quantitative, descriptive study, carried out with students from the physiotherapy course from the first to the last year of a public university in the interior of the Amazon. The WHOQOL-Bref questionnaire was used to assess the quality of life of these students. Results: Thirty students of both sexes from the 1st, 3rd and 5th year Physiotherapy course of a State University in the interior of the Amazon participated in the research, in which those who obtained the highest quality of life result were the 5th year students with a total of $57.21 \%$, in the second year with $52.88 \%$ and finally the 1 st year with $49,76 \%$. Conclusion: 1 st year students had the worst quality of life because they answered that they are dissatisfied with their physical and mental health, social relationships and the environment they live in.
\end{abstract}

Keywords: Quality of life, Universities, Mental health.

\section{RESUMEN}

Objetivo: Evaluar la calidad de vida de los estudiantes del curso de fisioterapia en una universidad pública del interior de la Amazonía y comparar los valores encontrados. Métodos: Estudio comparativo, transversal, cuantitativo, descriptivo, realizado con estudiantes del curso de fisioterapia del primero al último año de una

1 Universidade do Estado do Pará (UEPA), Santarém - PA. *E-mail: souzalorena511@gmail.com 
universidad pública del interior de la Amazonía. Se utilizó el cuestionario WHOQOL-Bref para evaluar la calidad de vida de estos estudiantes. Resultados: Participaron de la investigación 30 estudiantes de ambos sexos en el curso de Fisioterapia de $1^{\circ}, 3^{\circ}$ y $5^{\circ}$ año de una Universidad Estatal en el interior de la Amazonía, los estudiantes respondieron preguntas sobre los dominios: físico, psicológico, medio ambiente y relaciones sociales, en el que los que obtuvieron el mayor resultado en calidad de vida fueron los estudiantes de $5^{\circ}$ año con un total de $57.21 \%$, en el segundo año con $52.88 \%$ y finalmente el 1 er año con $49,76 \%$. Conclusión: Los estudiantes de 1er año tuvieron la peor calidad de vida porque respondieron que están insatisfechos con su salud física y mental, las relaciones sociales y el entorno en el que viven.

Palabras clave: Calidad de vida, Estudiantes universitarios, Salud mental.

\section{INTRODUÇÃO}

Conforme uma pesquisa realizada com o objetivo de definir o termo e se referir à avaliação da qualidade de vida, há indícios de que o termo apareceu pela primeira vez na literatura médica na década de 30 (SEIDL EMF, 2004). Segundo a Organização Mundial da Saúde (OMS) (2013), qualidade de vida é definida como a percepção dos indivíduos de sua integração na vida no contexto de sistemas culturais e de valores relacionados a seus objetivos, expectativas, padrões e preocupações. Está relacionada à saúde física, mental, grau de independência, relações sociais e relações com o ambiente pessoal características.

O momento atual é marcado pelo aumento das atividades cotidianas e diminuição do tempo, tanto para realizá-las como para o lazer. O indivíduo acaba buscando executá-las com êxito, e o aumento da competitividade faz com que sejam pessoas sobrecarregadas, ocupadas e pressionadas. Devido á busca por maior produtividade, maior conhecimento, pela ânsia de ter mais e saber mais, o indivíduo acaba deixando seu bem-estar e consequentemente sua qualidade de vida para segundo plano (FIEDLER PT, 2008).

No ambiente das universidades, a qualidade de vida dos acadêmicos tem relação direta com os acontecimentos da vida pessoal e acadêmica, como problemas que envolvam família e estado de saúde, questões relacionadas ao financeiro, busca da independência e futuro profissional, de modo que passa a ter ligação direta, de forma negativa, com o bem-estar ambiental, psíquico, físico e social, além de refletir na aprendizagem, autoestima e na formação profissional (SILVA PCS, et al., 2019).

De acordo com o estudo de Silva PCS, et al. (2019), os acadêmicos principalmente da área da saúde, apresentam altos níveis de estresse e ansiedade, que acarretam prejuízos na qualidade de vida e no desempenho do indivíduo. Os estudos também demonstram que os acadêmicos apresentam níveis de estresse superior a população saudável. Cada vez crescem o número de pessoas com doenças psicológicas, como a depressão, ansiedade por isso é de suma importância ter uma atenção voltada para estes futuros profissionais, pois é necessário estar bem consigo mesmo para poder fazer o melhor trabalho com as pessoas.

Além disso, estudos têm evidenciado que variáveis sociodemográficas como sexo, idade e escolaridade interferem na qualidade de vida. Indivíduos adultos jovens apresentam maior média para o domínio de relações sociais (NAHAS MV, 2013; VALINOTE HC, 2014; PALHARES VC, et al., 2014). Outro fator que tem papel importante na qualidade de vida é o sono. De acordo com Marconato RS, Monteiro MI (2015), existe uma associação direta entre horas de sono e domínio físico.

No qual um dos instrumentos para avaliar a qualidade de vida é o World Health Organization Quality of Life (WHOQOL-100), inicialmente tinham 100 questões, no seu desenvolvimento teve a participação de vários países, porém o Grupo de Qualidade de Vida da Organização Mundial de Saúde (OMS), decidiu desenvolver uma versão abreviada, o WHOQOL-bref, para que o questionário se tornasse rápido e fácil de ser aplicado com características psicométricas satisfatórias. Esse instrumento é composto por 4 domínios: físico, psicológico, relações sociais e meio ambiente (FLECK MPA, et al., 1999).

Diante do exposto, torna-se fundamental estudar a qualidade de vida no ambiente universitário, uma vez que a avaliação da segurança física, mental, social, da saúde e do bem-estar permite identificar a satisfação 
pessoal do discente, o que interfere na qualidade, no desenvolvimento e também na produtividade do trabalho acadêmico. Assim, o presente estudo busca avaliar a qualidade de vida e seus fatores associados em discentes de uma Universidade do interior da Amazônia.

\section{MÉTODOS}

A pesquisa seguiu a resolução № 466, de 12 de novembro de 2012, art. 3ํ, do Ministério da Saúde, em que fala dos aspectos éticos da pesquisa envolvendo seres humanos: respeito à autonomia, aos valores culturais, sociais, éticos, religiosos, hábitos e costumes dos participantes da pesquisa. Além disso, foi submetida à avaliação pelo Comitê de Ética em Pesquisa com o parecer de aprovação de número CAAE 4.464.058.

O presente estudo se caracteriza dentro de um método comparativo, pois tem como objetivo comparar a qualidade de vida dos discentes do curso de fisioterapia, e determinar qual turma tem a melhor e qual tem a pior qualidade de vida. Além disso, é uma pesquisa do tipo quantitativa, pois coletou dados do questionário de qualidade de vida e os transformou em números para assim serem tabulados e analisados. Outrossim, também é do tipo descritivo, visto que apresentou e analisou o resultado do fenômeno da qualidade de vida dos discentes do curso de fisioterapia.

O estudo também é considerado transversal, em que o pesquisador realiza a coleta dos dados do experimento em um ou mais momentos, ocorrendo um acompanhamento do fenômeno em estudo por um período de tempo (JUNG CF e ENG M, 2003). A presente pesquisa se enquadra nessa descrição, pois analisou apenas um momento no tempo para coletas dos dados e análises.

Foram incluídos os discentes devidamente matriculados no $1^{\circ}, 3^{\circ}$ e $5^{\circ}$ ano do curso de fisioterapia de uma Universidade pública do interior da Amazônia, maiores de 18 anos, que estivessem cursando há no mínimo 6 meses o curso. Empregaram-se como critérios de exclusão, discentes com dependência nos semestres anteriores, além dos que não preencheram de forma adequada o questionário e que fazem mais de uma graduação.

A coleta de dados da pesquisa ocorreu por meio de um questionário, o instrumento WHOQOL-bref que ocorreu de forma online, através do Google Forms. O WHOQOL-bref é um instrumento que avalia a qualidade de vida em quatro domínios, sendo eles: físico, psicológico, relações sociais e ambientais. Além disso, o valor de cada domínio varia de um 1 a 5 , sendo que quanto maior a pontuação, maior a qualidade de vida.

No domínio físico estão englobadas questões referentes a atividades de vida diária, dependência de substâncias medicinais e ajuda médica, energia e fadiga, mobilidade, dor e desconforto, sono e repouso, e capacidade para o trabalho. O domínio psicológico inclui imagem corporal e aparência, sentimentos positivos e negativos, autoestima, espiritualidade/religião/crenças pessoais, pensamento, aprendizagem, memória e concentração. No domínio relações sociais estão incorporadas questões sobre relações pessoais, suporte social e atividade sexual.

Por fim, o domínio ambiente inclui recursos financeiros, liberdade, segurança, cuidado social e de saúde (acesso e qualidade), ambiente domiciliar, oportunidades para adquirir novas informações e habilidades, participação e oportunidades de recreação/atividade de lazer, ambiente físico (poluição/barulho/tráfego/clima) e transporte (FLECK MPA, et al., 1999).

\section{RESULTADOS}

Participaram da pesquisa 30 discentes do curso de fisioterapia, de ambos os sexos, e que estivessem cursando o $1^{\circ}, 3^{\circ}$ ou $5^{\circ}$ ano do curso de fisioterapia. Ademais, através do questionário foi possível observar a qualidade de vida dos discentes de cada ano proposto.

Como verificado na Figura 1, os discentes do $1^{\circ}$ ano têm sua maior porcentagem no domínio de meio ambiente, apresentando $55,44 \%$, seguido pelas relações pessoais com $52,08 \%$, já no domínio psicológico apontam 50,52\%, e por último o domínio físico que denotam 40,18\%. Diante disso, devido a porcentagem em 
cada domínio foi possível indicar o valor total da qualidade de vida desses discentes sendo a porcentagem de 49,76\%, de acordo com a avaliação do questionário WHOQOL-Bref.

Figura 1 - Demonstra a porcentagem da qualidade de vida dos discentes do 1 ํano do curso de fisioterapia.

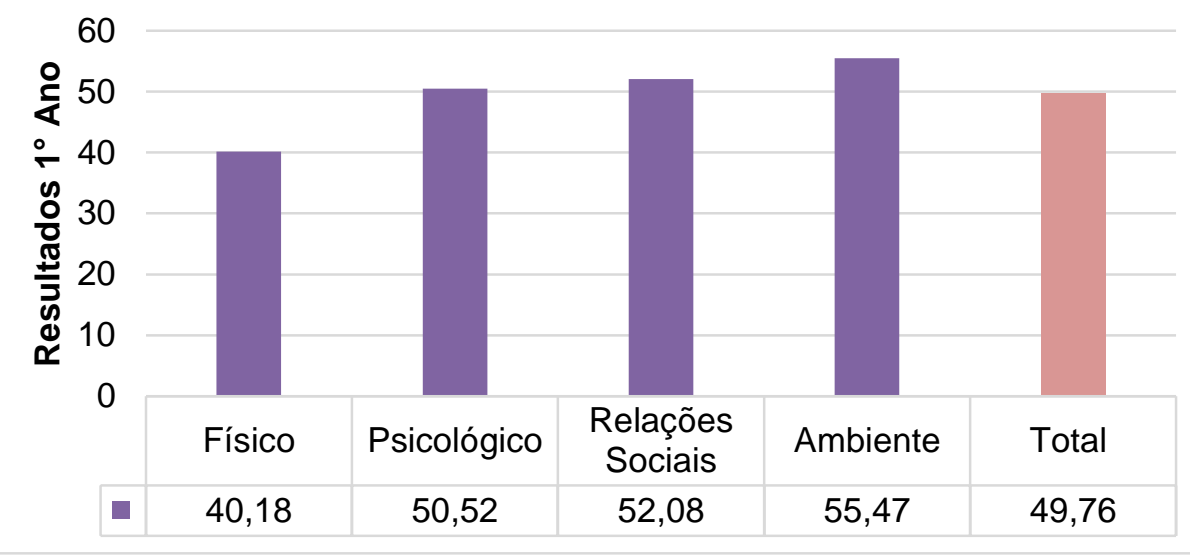

Nota: Taxa da qualidade de vida dos discentes de fisioterapia do $1^{\circ}$ ano.

Fonte: Silva LMS, et al., 2021.

Como demonstrado na Figura 2, os discentes do $3^{\text {o }}$ ano têm sua maior porcentagem no domínio de relações sociais, apresentando $58,25 \%$, seguido pelo domínio psicológico com $56,25 \%$, já no ambiente apontam $55,27 \%$, e por último o domínio físico que denotam $44,42 \%$. Assim sendo, devido a porcentagem em cada domínio foi possível indicar o valor total da qualidade de vida desses discentes com a porcentagem de $52,88 \%$, de acordo com a avaliação do questionário WHOQOL-Bref.

Figura 2 - Demonstra a porcentagem da qualidade de vida dos discentes do $3^{\circ}$ ano do curso de fisioterapia.

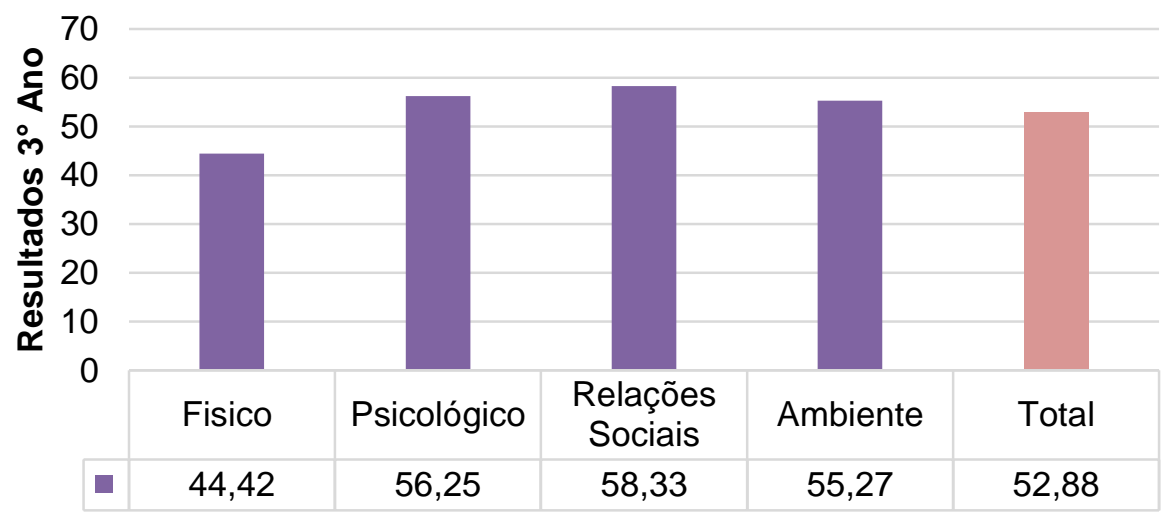

Nota: Taxa da qualidade de vida dos discentes de fisioterapia do $3^{\circ}$ ano.

Fonte: Silva LMS, et al., 2021.

Como verificado na Figura 3, os discentes do $5^{\circ}$ ano têm sua maior porcentagem no domínio psicológico, apresentando $61,81 \%$, seguido pelo ambiente com $59,9 \%$, já no físico apontam $54,17 \%$, e por último as relações sociais que denotam $54,17 \%$. Sendo assim, devido a porcentagem em cada domínio foi possível indicar o valor total da qualidade de vida desses discentes com a porcentagem de $57,21 \%$ de acordo com a avaliação do questionário WHOQOL-Bref. 
Figura 3 - Demonstra a porcentagem da qualidade de vida dos discentes do $5^{\circ}$ ano do curso de fisioterapia.

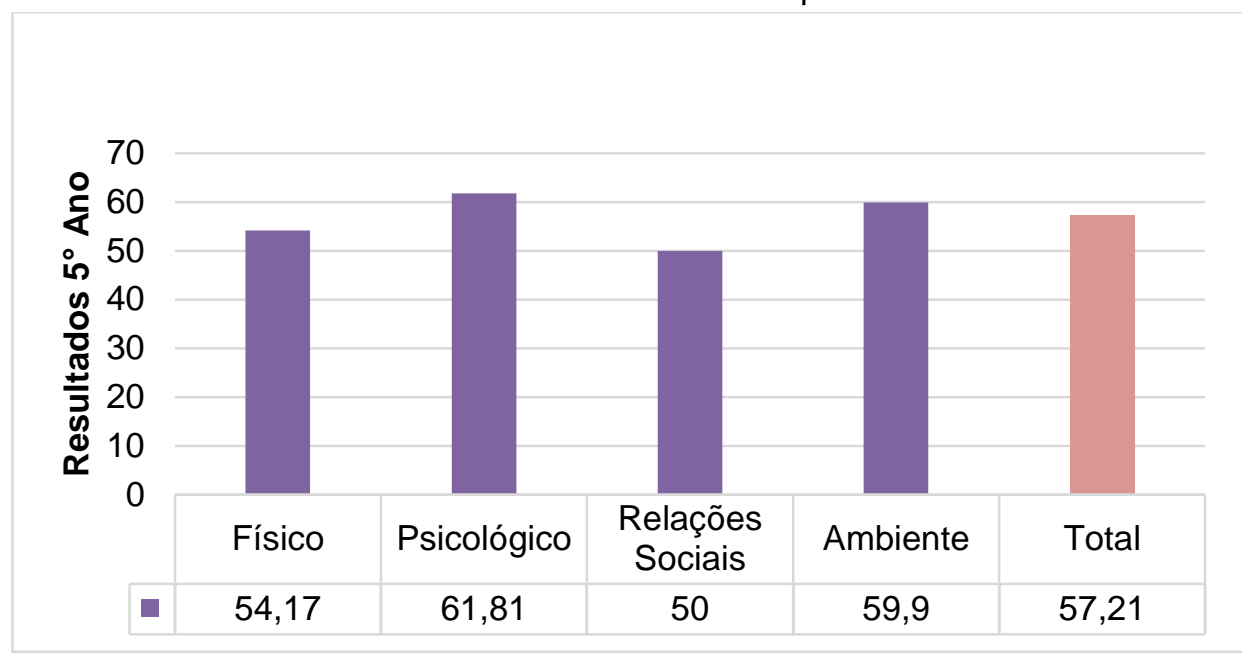

Nota: Taxa da qualidade de vida dos discentes de fisioterapia do $5^{\circ}$ ano.

Fonte: Silva LMS, et al., 2021.

De acordo com a Tabela 1, é possível verificar que os discentes do $1^{\circ}$ ano possuem a pior qualidade de vida com $49,76 \%$ enquanto os discentes do $5^{\circ}$ ano denotam a melhor qualidade de vida com $57,21 \%$. Além disso, os discentes do $3^{\circ}$ ano apresentaram uma qualidade de vida mediana com $52,88 \%$.

Tabela 1 - Qualidade de vida dos discentes de fisioterapia do $1^{\circ}, 3^{\circ}$ e e $5^{\circ}$ ano.

\begin{tabular}{cc}
\hline Ano & Média \\
\hline $1^{\circ}$ & $49,76 \%$ \\
$3^{\circ}$ & $52,88 \%$ \\
$5^{\circ}$ & $57,21 \%$ \\
\hline
\end{tabular}

Fonte: Silva LMS, et al., 2021.

\section{DISCUSSÃO}

O termo qualidade de vida pode estar relacionado com o funcionamento diário do indivíduo, que pode afetar os sentimentos, comportamentos e a percepção. A interpretação da qualidade de vida é bem complexa, pois ela se distingue de acordo com a cultura, época e indivíduo, e pode acontecer de se mudar o conceito de qualidade de vida num mesmo indivíduo, por exemplo, o que ele acha que é uma boa qualidade de vida hoje, pode não ser a mesma daqui alguns meses (PRAÇA MIF, 2012).

A qualidade de vida dos discentes está diretamente relacionada à vida pessoal e acadêmica, como questões relacionadas à família e às condições de saúde, questões relacionadas às finanças e questões de busca de independência e perspectivas de carreira, portanto, a qualidade de vida dos discentes está diretamente relacionada com o bem-estar ambiental, psíquico, físico e social, e também reflete na autoestima, aprendizagem e formação profissional (DE SOUSA SILVA PC, et al., 2019).

Com a análise dos resultados alcançados, foi possível constatar que os discentes do $1^{\circ}$ ano do curso de fisioterapia possuem a pior qualidade de vida, em comparação com os outros grupos analisados, isso ocorre devido ao contato inicial com o âmbito universitário ser um novo ambiente com muitas diferenças comparadas ao ensino médio, com muitas adaptações a nova moradia, novas responsabilidades, novas formas de ensino e com as muitas novidades podem ocorrer muitas mudanças e adaptações pelas quais o universitário terá que se adequar.

De acordo com o estudo de De Sousa Silva PC, et al. (2019), os discentes de fisioterapia sofrem problemas relacionados à saúde, o que prejudica diretamente a sua qualidade de vida, interferindo em seu rendimento 
acadêmico, além disso foi possível verificar que nenhum dos discentes que participaram da pesquisa possuem algum transtorno mental, que possa prejudicar o seu processo de aprendizagem. Ademias, esse estudo concorda com a pesquisa em questão, pois fala dos problemas relacionadas à saúde dos discentes de fisioterapia, e foi possível observar na presente pesquisa, que a menor pontuação foi com o domínio físico do indivíduo, ou seja, com a sua saúde, afetando diretamente o seu rendimento acadêmico.

As adaptações e mudanças podem interferir no estado fisiológico e comportamental do indivíduo, de acordo com Carvalho TMCS, et al. (2013) durante o sono ocorrem mudanças fisiológicas e comportamentais que interferem diretamente no processo cognitivo e de aprendizagem. Os universitários que acabaram de ser admitidos na universidade se deparam com elevados requisitos acadêmicos e horários irregulares de aulas, o que acarreta na mudança do seu padrão de ciclo sono-vigília para a realização das atividades acadêmicas.

Pessoas que sofrem de privação de sono são mais fracas em produtividade e ambição. Além disso, a falta de sono também pode alterar o desempenho de habilidades cognitivas, como memória, raciocínio lógico, capacidade de cálculo e capacidade de tomada de decisão. As atividades diárias dos universitários nem sempre são acompanhadas de hábitos de sono saudáveis, Além de que mudanças nos padrões de sonovigília podem levar à hipertensão e obesidade, também como pode levar à redução da qualidade de vida, encurtamento do tempo de vida e envelhecimento prematuro.

Ademais, uma característica apresentada por muitos acadêmicos é o estresse, estudos mostram que estudantes que apresentam uma dupla jornada, trabalho e estudo universitário têm um nível de estresse muito mais elevado do que aqueles que somente estudam. Tendo assim um maior nível de estresse e uma menor qualidade de vida. No qual o estresse costuma afetar o indivíduo em seu comportamento e, em longo prazo traz consequências negativas para a qualidade de vida, incluindo o campo fisiológico, psicológico e comportamental. Podendo gerar ansiedade, atitudes negativas em relação ao desempenho causando frustração e redução da produtividade (MARCHINI DMF, et al, 2019).

Níveis de estresse elevados podem causar danos prejudiciais para o estudante universitário, ele é resultante de um conjunto de reações fisiológicas e comportamentais que irão alterar a homeostasia do organismo. Gerando respostas como consequência desses estímulos, dentre eles a ansiedade. $O$ estresse causado por essa fase na universidade é contribuinte para o surgimento da ansiedade, que pode ser apresentada de forma aguda ou crônica, é um estado emocional que acompanha uma série de alterações comportamentais e psicológicas. É um dos principais fatores que podem causar prejuízo no desempenho dos estudos (LANTYER AS, et al., 2016).

Outrossim, de acordo com a Tabela 1 também foi possível observar que o $5^{\circ}$ ano apresenta a melhor qualidade de vida, isso acontece adaptaram-se à sua rotina e com a mente mais madura e focada no seu futuro profissional, com matérias mais na vivência prática com pacientes, menos contato somente com a sala de aula, sendo assim, conseguem ter uma vida social melhor, influenciando diretamente na sua qualidade de vida.

Ainda assim, no estudo de Catunda MAP e Ruiz VM (2008) foi possível constatar que a qualidade de vida dos discentes que estão cursando os últimos anos são melhores comparada com a qualidade de vida dos que cursam o $1^{\circ}$ ano, isso se deve ao fato de que os universitários dos últimos anos estão mais adaptados à rotina universitária. Esse estudo concorda com a presente pesquisa, em que os resultados foram que os discentes do $5^{\circ}$ ano possuem uma melhor qualidade de vida em comparação com o $1^{\circ}$ ano.

Além disto, a qualidade de vida vai muito além da condição física, abrange também o psicológico e o social (PRAÇA MIF, 2012). Segundo muitos autores, qualidade vida seria o sinônimo de saúde, mas para outros seria um conceito mais abrangente, em que a condição de saúde seria um aspecto considerado para a qualidade de vida (PEREIRA EF, et al., 2012).

Além disso, de acordo com a Tabela 1 constatou-se que os discentes do $3^{\circ}$ ano possuem uma qualidade de vida mediana, isso ocorre devido estarem na metade do curso, por isso acabam tendo características de qualidade de vida tanto do $1^{\circ}$ ano quanto do $5^{\circ}$. Ademais, não possuem a melhor qualidade de vida, pois estão se aproximando do fim do curso e começam a se preocupar com várias situações profissionais. 
A qualidade de vida possui um conceito amplo incluindo o estado psicológico, saúde física, relações sociais, nível de independência, crenças pessoais, entre outros aspectos, então sua percepção sobre sua saúde pode influenciar a percepção sobre qualidade de vida do universitário (ROSA MJ, et al., 2019).

A falta de qualidade de vida pode causar déficit na saúde mental por também trazer uma pressão por aprender muitas informações do curso, pouco tempo de atividade de lazer, contado com estudo de doenças no caso de estudantes da área da saúde, e uma tensão maior causada pelas atividades da universidade (BUHRER BE, et al., 2019).

Pode ser encontrado diversos problemas musculares causados muitas vezes pela má postura, principalmente na hora dos estudos, o que pode ocasionar dor e desconforto, além de prejudicar a realização das atividades da vida cotidiana. Além disso, alguns discentes acabam tendo uma qualidade de sono ruim, o que afeta diretamente a sua energia, causando uma diminuição na sua capacidade de trabalho. Muitos desses discentes devido a correria da vida diária, a algumas cobranças excessivas e preocupação com a carreira profissional, se tornam dependentes de medicamentos e de tratamentos psicológicos e/ou psiquiátricos. Os problemas musculares nos universitários também podem ser causados em decorrência do longo tempo na postura sentada, pela presença de tensão muscular, dores da costa, patologias na coluna vertebral, causando até limitações no movimento e alterações na funcionalidade (BACCHI CA, et al., 2013).

O consumo de álcool por universitários está relacionado com a qualidade de vida, o álcool é uma das formas dos acadêmicos usar como distração e relaxamento, é uma substancia psicoativa que pode trazer diversos danos, estudos mostraram que acadêmicos que tem uma baixa qualidade de vida bebem, e isso diminui a vitalidade, alterações nos aspectos emocionais, diminuição da saúde mental, estado geral de saúde (SAWICKI WC, et al., 2017).

A ingestão de bebidas alcoólicas pelos universitários se torna cada dia mais preocupante, pois geralmente são consumidas várias quantidades de álcool em uma única ocasião. Além disso, existe a falta de programas educativos, voltados para a conscientização desses jovens em relação ao consumo inadequado do álcool, sendo importante mostrá-los os problemas que a ingestão de álcool exagerada pode ocasionar, sendo que a dependência alcoólica é um dos principais problemas médicos, atingindo tanto homens quanto mulheres (SILVA JN, et al., 2016).

Outro fator que pode estar associado a perda da qualidade de vida é o fator financeiro, pois muitos dos discentes ainda estão na dependência financeiras dos pais porém morando sozinhos, longe dos lares familiares, tendo gastos com transportes, materiais de estudo, bem como alimentação, aluguel e gastos de moradia, com essa nova responsabilidade financeira, possivelmente não tenham a opção dos gastos com o lazer.

No estudo de Silva EC e Heleno MGV (2012) no qual também foi utilizado o instrumento WHOQOL-bref, para a avaliação da qualidade de vida dos universitários, foi possível constatar que o domínio relações socais apresentou a maior média, seguido pelo domínio físico, psicológico e domínio meio ambiente. Se contrapondo ao estudo em execução, que a ordem dos domínios variou conforme o ano de estudo dos universitários, em que nos universitários do $1^{\circ}$ ano a maior média foi no domínio ambiente, seguida do domínio relações sociais, psicológico e físico, já nos universitários do $3^{\circ}$ ano, a maior média apresentada no domínio relações sociais, seguido do domínio psicológico, ambiente e físico, e por último, nos universitários do 5aao, a maior média foi no domínio psicológico, seguido pelo ambiente, físico e relações sociais.

\section{CONCLUSÃO}

Os resultados do presente estudo revelam que os discentes do $1^{\circ}$ ano apresentam uma pior qualidade de vida, isso ocorre devido estarem iniciando o curso, aprendendo conteúdos novos e se adaptando a uma nova rotina, sendo sugerida uma atenção voltada para esse público, com o objetivo de diminuir os impactos sobre a sua qualidade de vida. Ademais, os discentes do $5^{\circ}$ ano apresentam uma melhor qualidade de vida, pois já se adaptaram as dificuldades do curso. Uma das fragilidades do estudo, está relacionada ao quantitativo, porém devido ao momento vivido no país, o enfrentando da pandemia da COVID-19, isso pode ter interferido diretamente para que alguns discentes não participassem da pesquisa. 


\section{REFERÊNCIAS}

1. ARIÑO DO, BARDAGI MP. Relação entre fatores acadêmicos e a saúde mental de estudantes universitários. Revista Psicologia em Pesquisa, 2018; 12(3).

2. BORGES MR, et al. Comportamento sexual de ingressantes universitários. Revista de pesquisa cuidado é fundamental online, 2015; 7(2): 2505-2515.

3. BUHRER BE, et al. Análise da Qualidade e Estilo de Vida entre Acadêmicos de Medicina de uma Instituição do Norte do Paraná. Rev. Bras. Educ. Med. Brasília, 2019; 43(1): 39-46.

4. BACCHI CA, et al. Avaliação da qualidade de vida, da dor nas costas, da funcionalidade e de alterações da coluna vertebral de estudantes de fisioterapia. Motriz: Rev. Educ. Fis. Rio Claro, 2013; 19(2): 243-251.

5. CARVALHO TMCS, et al. Qualidade do sono e sonolência diurna entre estudantes universitários de diferentes áreas. Revista Neurociências, 2013; 21(3): 383-387.

6. CATUNDA MAP, RUIZ VM. Qualidade de vida de universitários. Pensamento plural, 2008; 2(1): 22-31.

7. JUNG CF, ENG M. Metodologia científica. Ênfase em pesquisa tecnológica, 2003; 3 (41) v. 3, p. 41.

8. DA SILVA LANTYER A, et al. Ansiedade e qualidade de vida entre estudantes universitários ingressantes: avaliação e intervenção. Revista Brasileira de Terapia Comportamental e Cognitiva, 2016; 18(2): 4-19.

9. DE SOUSA SILVA PC, et al. Qualidade de vida e condições de saúde de acadêmicos do curso de fisioterapia. Revista Brasileira de Educação e Saúde, 2019; 9(4): 10-17.

10. FIEDLER PT. Avaliação da qualidade de vida do estudante de medicina e da influência exercida pela formação acadêmica. São Paulo, 2008.

11. DEL CIAMPO LA Antonio, DEL CIAMPO IRL. Adolescência e imagem corporal. Adolescência e Saúde, 2010; 7(4): 55-59.

12. FONTELLES MJ, et al. Metodologia da pesquisa científica: diretrizes para a elaboração de um protocolo de pesquisa. Revista Paraense De Medicina, 2009; 23(3): 1-8.

13. FLECK MPA. O instrumento de avaliação de qualidade de vida da Organização Mundial da Saúde (WHOQOL-100): características e perspectivas. Ciência \& Saúde Coletiva, 2000; 5: 33-38.

14. FLECK MPA, et al. Desenvolvimento da versão em português do instrumento de avaliação de qualidade de vida da OMS (WHOQOL-100). Brazilian Journal of Psychiatry, 1999; 21(1): 19-28.

15. GIL AC. Métodos e técnicas de pesquisa social. 6. ed. Ediitora Atlas SA, 2008.

16. MARCHINI DMF, et al. Análise de Estresse e Qualidade de Vida em Alunos Universitários. Revista de Administração Unimep, 2019; 17 (3): 141-164.

17. MARCONATO RS, MONTEIRO MI. Dor, percepção de saúde e sono: impacto na qualidade de vida de bombeiros/profissionais do resgate. Revista Latino-Americana de Enfermagem, 2015; 23 (6), 991-999.

18. NAHAS MV. Atividade física, saúde e qualidade de vida: conceitos e para um estilo de vida ativo. Midiograf, 2006.

19. PALHARES VC, et al. Association between sleep quality and quality of life in nursing professionals working rotating shifts. Revista de Saúde Pública, 2014; 48: 594-601.

20. PEREIRA EF, et al. Qualidade de vida: abordagens, conceitos e avaliação. Revista Brasileira de Educação Física e Esporte, 2012; 26 (2): 241-250.

21. PRAÇA FSG. Metodologia da pesquisa científica: organização estrutural e os desafios para redigir o trabalho de conclusão. Revista Eletrônica "Diálogos Acadêmicos, 2015; 8 (1): 72-87.

22. PRAÇA MIF. Qualidade de vida relacionada com a saúde: a perspectiva dos utentes que frequentam os Centros de Saúde do ACES Trás-os-Montes I Nordeste. 2012. Tese de Doutorado. Instituto Politécnico de Bragança, Escola Superior de Tecnologia e Gestão.

23. ROSA MJ, et al. Nível de atividade física, qualidade de vida e fatores associados de estudantes universitários de Educação Física. RBPFEX-Revista Brasileira de Prescrição e Fisiologia do Exercício, 2019; 13 (81): 86-91.

24. SAWICKI WC, et al. Consumo de álcool, qualidade de vida, Intervenção Breve entre universitários de Enfermagem. Revista Brasileira de Enfermagem, 2018; 71: 505-512.

25. SEIDL EMF, ZANNON CMLC. Qualidade de vida e saúde: aspectos conceituais e metodológicos. Cadernos de saúde pública, 2004; 20: 580-588.

26. SILVA JN, et al. Consumo álcool entre universitários. Revista Brasileira de Pesquisa em Ciências da Saúde, 2016 ; 2 (2): 35-40.

27. SILVA EC, HELENO MGV. Qualidade de vida e bem-estar subjetivo de estudantes universitários. Revista Psicologia e Saúde, 2012.

28. SILVA PCS, et al. Qualidade de vida e condições de saúde de acadêmicos do curso de fisioterapia. Revista Brasileira de Educação e Saúde, 2019; 9 (4): 10-17.

29. TEIXEIRA MAP, et al. Adaptação à universidade em jovens calouros. Psicologia escolar e educacional, 2008; 12(1): 185-202.

30. VALINOTE HC, et al. Análise da qualidade de vida, capacidade para o trabalho e nível de estresse em trabalhadores da construção civil. Revista Brasileira de Ciências Ambientais (Online), 2014; 32: 115-126.

31. WORLD HEALTH ORGANIZATION. WHOQOL-BREF Introduction, administration, scoring and generic version of the assessment [Internet]. Geneva, 1996. 